\title{
SCHUR TENSOR PRODUCT OF OPERATOR SPACES
}

\author{
VANDANA RAJPAL, AJAY KUMAR ${ }^{1}$ AND TAKASHI ITOH
}

\begin{abstract}
We develop a systematic study of the schur tensor product both in the category of operator spaces and in that of $C^{*}$-algebras.
\end{abstract}

\section{INTRODUCTION}

An operator space is a closed subspace of the space $B(H)$ of all bounded operators on a Hilbert space $H$. The fundamental and systematic developments in the theory of tensor product of operator spaces have been evolved considerably, see e.g. 4], 5]. In this category, the Haagerup tensor norm is the natural one for compatibility with the continuity of the completely bounded bilinear maps, and the operator space projective tensor norm is for the jointly completely bounded bilinear maps. For operator spaces $V$ and $W$, and elements $x=\left[x_{i j}\right] \in M_{n}(V)$ and $y=\left[y_{i j}\right] \in M_{n}(W)$, we define an element $x \circ y \in M_{n}(V \otimes W)$ by $x \circ y=\left[x_{i j} \otimes y_{i j}\right]$. Note that $x \circ y=\left[e_{11}, e_{22}, e_{33}, \cdots, e_{n n}\right](x \otimes y)\left[e_{11}, e_{22}, e_{33}, \cdots, e_{n n}\right]^{t}$, where $x \otimes y$ denotes the kronecker tensor product, and $\left\{e_{i j}\right\}$ are the standard basis of $M_{n}, n \in \mathbb{N}$. Each element $u$ in $M_{p}(V \otimes W), p \in \mathbb{N}$, can be written as $u=\alpha(x \circ y) \beta$ for some $x \in M_{n}(V), y \in M_{n}(W), \alpha \in M_{p, n}$, and $\beta \in M_{n, p}$, $n \in \mathbb{N}$, and we define

$$
\|u\|_{s}=\inf \{\|\alpha\|\|x\|\|y\|\|\beta\|\}
$$

where infimum is taken over arbitrary decompositions as above. Let $V \otimes_{s}$ $W=\left(V \otimes W,\|\cdot\|_{s}\right)$, and define the schur tensor product $V \otimes^{s} W$ to be the completion of $V \otimes W$ in this norm. In Section 2 we look at the schur tensor norm in the context of operator spaces, and show that this is an operator space matrix norm. We also introduce the notion of schur bounded bilinear maps and show that the schur tensor norm may be used to linearize them. This parallel development of the theory of schur tensor product of operator spaces will play a vital role in the theory of operator spaces. In analogy to the operator space projective tensor product, the schur tensor product turns out to be commutative, projective, and functorial however we don't know whether is it associative or not. At the end of this section, we define a new tensor norm, which we denote by $\|\cdot\|_{s^{\prime}}$, and it will be seen that the dual of

1. corresponding author 2010 Mathematics Subject Classification. Primary 46L06, Secondary 46L07,47L25.

Key words and phrases. Schur tensor norm, schur bounded bilinear maps. 
the schur tensor norm is in fact $\|\cdot\|_{s^{\prime}}$-norm. Section 3 is concerned with the equivalence of the schur tensor norm with the various other norms.

Recall that the operator space projective tensor norm on the algebraic tensor product of two operator spaces $V$ and $W$ is defined as, for $u \in V \otimes W$, $\|u\|_{\wedge}=\inf \{\|\alpha\|\|x\|\|y\|\|\beta\|\}$, the infimum is taken over $p, q \in \mathbb{N}$ and all the ways to write $u=\alpha(x \otimes y) \beta$, where $\alpha \in M_{1, p q}, \beta \in M_{p q, 1}, x \in M_{p}(V)$ and $y \in M_{q}(W)$, and $x \otimes y=\left(x_{i j} \otimes y_{k l}\right)_{(i, k),(j, l)} \in M_{p q}(V \otimes W)$. The operator space projective tensor product $V \widehat{\otimes} W$ is defined to be the completion of $V \otimes W$ in the norm $\|\cdot\|_{\wedge}[4]$. The jointly completely bounded norm of a bilinear map $\phi: V \times W \rightarrow Z$ is defined to be $\|\phi\|_{j c b}=\sup \left\{\left\|\phi_{(n)}\right\|: n \in \mathbb{N}\right\}$, where $\phi_{(n)}: M_{n}(V) \times M_{n}(W) \rightarrow M_{n^{2}}(Z)$ is given by $\phi_{(n)}\left(\left[v_{i j}\right],\left[w_{k l}\right]\right)=$ $\left(\phi\left(v_{i j}, w_{k l}\right)\right)$.

\section{SCHUR TENSOR PRODUCT OF OPERATOR SPACES}

Theorem 2.1. For operator spaces $V$ and $W,\|\cdot\|_{s}$ is an operator space matrix norm on $V \otimes W$.

Proof: Given $u_{1} \in V \otimes W, u_{2} \in V \otimes W$ and $\epsilon>0$, choose $\alpha_{1} \in M_{1, r}$, $x_{1} \in M_{r}(V), y_{1} \in M_{r}(W), \beta_{1} \in M_{r, 1}$, and $\alpha_{2} \in M_{1, p}, x_{2} \in M_{p}(V), y_{2} \in$ $M_{p}(W), \beta_{2} \in M_{p, 1}, p, r \in \mathbb{N}$ such that $u_{1}=\alpha_{1}\left(x_{1} \circ y_{1}\right) \beta_{1}, u_{2}=\alpha_{2}\left(x_{2} \circ y_{2}\right) \beta_{2}$ and $\left\|\alpha_{1}\right\|\left\|x_{1}\right\|\left\|y_{1}\right\|\left\|\beta_{1}\right\|<\left\|u_{1}\right\|_{s}+\epsilon,\left\|\alpha_{2}\right\|\left\|x_{2}\right\|\left\|y_{2}\right\|\left\|\beta_{2}\right\|<\left\|u_{2}\right\|_{s}+\epsilon$, we may assume that $\left\|x_{i}\right\|=\left\|y_{i}\right\|=1$ and $\left\|\alpha_{i}\right\|=\left\|\beta_{i}\right\| \leq\left(\left\|u_{i}\right\|_{s}+\epsilon\right)^{\frac{1}{2}}$, for $i=1,2$. Let $\alpha=\left[\begin{array}{ll}\alpha_{1} & \alpha_{2}\end{array}\right], \beta=\left[\begin{array}{ll}\beta_{1} & \beta_{2}\end{array}\right]^{t}, v:=x_{1} \oplus x_{2}$, and $w:=y_{1} \oplus y_{2}$. Then $v \circ w=\left[\begin{array}{cc}x_{1} & 0 \\ 0 & x_{2}\end{array}\right] \circ\left[\begin{array}{cc}y_{1} & 0 \\ 0 & y_{2}\end{array}\right]=\left[\begin{array}{cc}x_{1} \circ y_{1} & 0 \\ 0 & x_{2} \circ y_{2}\end{array}\right]$, and so $u_{1}+u_{2}=\alpha(v \circ w) \beta$. Now, by Ruan's axioms of operator spaces [6] and the $C^{*}$-identity, we have

$$
\begin{aligned}
\left\|u_{1}+u_{2}\right\|_{s} & \leq\|\alpha\|\|\beta\|, \\
& \leq \frac{1}{2}\left(\|\alpha\|^{2}+\|\beta\|^{2}\right), \\
& =\frac{1}{2}\left\|\left[\begin{array}{cc}
\alpha_{1} & \alpha_{2} \\
0 & 0
\end{array}\right]\right\|^{2}+\left\|\left[\begin{array}{cc}
\beta_{1} & 0 \\
\beta_{2} & 0
\end{array}\right]\right\|^{2} \\
& =\frac{1}{2}\left\|\left[\begin{array}{cc}
\alpha_{1} & \alpha_{2} \\
0 & 0
\end{array}\right]\left[\begin{array}{cc}
\alpha_{1}^{*} & 0 \\
\alpha_{2}^{*} & 0
\end{array}\right]\right\|+\left\|\left[\begin{array}{cc}
\beta_{1}^{*} & \beta_{2}^{*} \\
0 & 0
\end{array}\right]\left[\begin{array}{cc}
\beta_{1} & 0 \\
\beta_{2} & 0
\end{array}\right]\right\| \\
& =\frac{1}{2}\left\|\left[\begin{array}{cc}
\alpha_{1} \alpha_{1}^{*}+\alpha_{2} \alpha_{2}^{*} & 0 \\
0 & 0
\end{array}\right]\right\|+\left\|\left[\begin{array}{cc}
\beta_{1}^{*} \beta_{1}+\beta_{2}^{*} \beta_{2} & 0 \\
0 & 0
\end{array}\right]\right\| \\
& =\frac{1}{2}\left\|\alpha_{1} \alpha_{1}^{*}+\alpha_{2} \alpha_{2}^{*}\right\|+\left\|\beta_{1}^{*} \beta_{1}+\beta_{2}^{*} \beta_{2}\right\|, \\
& \leq \frac{1}{2}\left(\left\|\alpha_{1}\right\|^{2}+\left\|\alpha_{2}\right\|^{2}+\left\|\beta_{1}\right\|^{2}+\left\|\beta_{2}\right\|^{2}\right), \\
& \leq\left\|u_{1}\right\|_{s}+\left\|u_{2}\right\|_{s}+2 \epsilon .
\end{aligned}
$$

Since $\epsilon>0$ is arbitrary, so we have the subadditivity. For any scalar $c \in \mathbb{C}$, it is clear that $\|c u\|_{s}=|c|\|u\|_{s}$.

Let $u=\alpha(x \circ y) \beta \in V \otimes W, \alpha=\left[\alpha_{i}\right] \in M_{1, p}, \beta=\left[\beta_{j}\right] \in M_{p, 1}, x \in M_{p}(V)$,

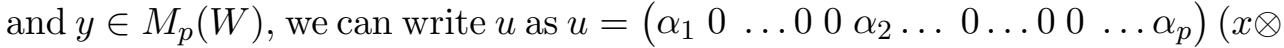
y) $\left(\beta_{1} 0 \ldots 00 \beta_{2} \ldots 0 \ldots 00 \ldots \beta_{p}\right)^{t}$, which is a representation in the set $\left\{\gamma(x \otimes y) \lambda: \gamma \in M_{1, p^{2}}, \lambda \in M_{p^{2}, 1}, x \in M_{p}(V), y \in M_{p}(W)\right\}$. Again, by Ruan's axioms and the $C^{*}$-identity, $\left\|\left(\begin{array}{lllllllllll}\alpha_{1} & 0 & \ldots & 0 & 0 & \alpha_{2} & \ldots & 0 & \ldots & 0 & 0\end{array} \ldots \alpha_{p}\right)\right\|=$ $\|\alpha\|$ and $\left\|\left(\begin{array}{lllllllllll}\beta_{1} & 0 & \ldots & 0 & 0 & \beta_{2} & \ldots & 0 & \ldots & 0 & 0\end{array} \beta_{p}\right)^{t}\right\|=\|\beta\|$. Hence $\|u\|_{\wedge} \leq$ $\|u\|_{s}$, which shows that $\|\cdot\|_{s}$ is a norm on $V \otimes W$. 
Finally, to see the Ruan's axioms. Suppose that $u_{1} \in M_{m}(V \otimes W)$, $u_{2} \in M_{n}(V \otimes W)$ and $\epsilon>0$, we may find decompositions, for $i=1,2$, $u_{i}=\alpha_{i}\left(x_{i} \circ y_{i}\right) \beta_{i}$ with $\left\|x_{i}\right\|=\left\|y_{i}\right\|=1$ and $\left\|\alpha_{i}\right\|=\left\|\beta_{i}\right\| \leq\left(\left\|u_{i}\right\|_{s}+\epsilon\right)^{\frac{1}{2}}$ as above, where $\alpha_{1} \in M_{m, r}, \alpha_{2} \in M_{n, s}, \beta_{1} \in M_{r, m}$, and $\beta_{2} \in M_{s, n}$. Let $v:=x_{1} \oplus x_{2}$ and $w:=y_{1} \oplus y_{2}$. Then $u_{1} \oplus u_{2}=\left[\begin{array}{cc}\alpha_{1} & 0 \\ 0 & \alpha_{2}\end{array}\right](v \circ w)\left[\begin{array}{cc}\beta_{1} & 0 \\ 0 & \beta_{2}\end{array}\right]$. So, by Ruan's axioms for $V$ and $W$, we have

$$
\left\|u_{1} \oplus u_{2}\right\|_{s} \leq\left\|\left[\begin{array}{cc}
\alpha_{1} & 0 \\
0 & \alpha_{2}
\end{array}\right]\right\|\left\|\left[\begin{array}{cc}
\beta_{1} & 0 \\
0 & \beta_{2}
\end{array}\right]\right\| \text {. }
$$

Let $t=\max \{m+n, r+s\}$. By adding rows and columns of zeros to matrices $\left[\begin{array}{cc}\alpha_{1} & 0 \\ 0 & \alpha_{2}\end{array}\right]$ and $\left[\begin{array}{cc}\beta_{1} & 0 \\ 0 & \beta_{2}\end{array}\right]$, we obtain the new matrices, say, $T_{1} \in M_{t}(\mathbb{C})$ and $T_{2} \in M_{t}(\mathbb{C})$ such that $\left[\begin{array}{cc}\alpha_{1} & 0 \\ 0 & \alpha_{2}\end{array}\right]$ and $\left[\begin{array}{cc}\beta_{1} & 0 \\ 0 & \beta_{2}\end{array}\right]$ are the upper left hand corner of $T_{1}$ and $T_{2}$, respectively, and so applying the $C^{*}$-identity, we have

$$
\begin{aligned}
\left\|u_{1} \oplus u_{2}\right\|_{s} & \leq\left\|\left[\begin{array}{cc}
\alpha_{1} \alpha_{1}^{*} & 0 \\
0 & \alpha_{2} \alpha_{2}^{*}
\end{array}\right]\right\|^{1 / 2}\left\|\left[\begin{array}{cc}
\beta_{1} \beta_{1}^{*} & 0 \\
0 & \beta_{2} \beta_{2}^{*}
\end{array}\right]\right\|^{1 / 2} \\
& =\left\|\alpha_{1} \alpha_{1}^{*} \oplus \alpha_{2} \alpha_{2}^{*}\right\|^{1 / 2}\left\|\beta_{1} \beta_{1}^{*} \oplus \beta_{2} \beta_{2}^{*}\right\|^{1 / 2} \\
& =\left(\max \left\{\left\|\alpha_{1}\right\|^{2},\left\|\alpha_{2}\right\|^{2}\right\}\right)^{1 / 2}\left(\max \left\{\left\|\beta_{1}\right\|^{2},\left\|\beta_{2}\right\|^{2}\right\}\right)^{1 / 2} \\
& \leq \max \left\{\left\|u_{1}\right\|_{s},\left\|u_{2}\right\|_{s}\right\}+\epsilon .
\end{aligned}
$$

Since $\epsilon$ is arbitrary, so $\left\|u_{1} \oplus u_{2}\right\|_{s} \leq \max \left\{\left\|u_{1}\right\|_{s},\left\|u_{2}\right\|_{s}\right\}$. Now let $\gamma \in$ $M_{p, m}$ and $\delta \in M_{m, p}$, then $\gamma u_{1} \delta=\left(\gamma \alpha_{1}\right)\left(x_{1} \circ y_{1}\right)\left(\beta_{1} \delta\right)$, and so $\left\|\gamma u_{1} \delta\right\|_{s} \leq$ $\left\|\gamma \alpha_{1}\right\|\left\|\beta_{1} \delta\right\| \leq\|\gamma\|\|\delta\|\left(\left\|u_{1}\right\|_{s}+\epsilon\right)$. Because $\epsilon$ was arbitrary, $\left\|\gamma u_{1} \delta\right\|_{s} \leq$ $\|\gamma\|\|\delta\|\left\|u_{1}\right\|_{s}$. Hence the result follows from ( [6], Proposition 2.3.6).

TheOREm 2.2. For operator algebras $X$ and $Y, X \otimes^{s} Y$ is a Banach algebra, and it is ${ }^{*}$-algebra provided both $X$ and $Y$ have isometric involution. Furthermore, if $X$ and $Y$ are approximately unital then $X \otimes^{s} Y$ has a bounded approximate identity.

Proof: Let $u, v \in X \otimes Y$ with $u=\sum_{i, j=1}^{n} \alpha_{i}\left(x_{i j} \otimes y_{i j}\right) \beta_{j}$, and $v=$ $\sum_{k, l=1}^{m} \gamma_{k}\left(z_{k l} \otimes w_{k l}\right) \delta_{l}$. Then $u v=\sum_{i, j, k, l} \alpha_{i} \gamma_{k}\left(x_{i j} z_{k l} \otimes y_{i j} w_{k l}\right) \beta_{j} \delta_{l}$, which can be further written as $u v=\left[\alpha_{i} \gamma_{k}\right]\left[x_{i j} z_{k l}\right] \circ\left[y_{i j} w_{k l}\right]\left[\beta_{j} \delta_{l}\right]$, and so $\|u v\|_{s} \leq\left\|\left[\alpha_{i} \gamma_{k}\right]\right\|\left\|\left[x_{i j} z_{k l}\right]\right\|\left\|\left[y_{i j} w_{k l}\right]\right\|\left\|\left[\beta_{j} \delta_{l}\right]\right\|$.

Thus, by ( [6], Proposition 2.1.1) and the fact that the operator algebras are completely contractive, we have

$\|u v\|_{s} \leq\left\|\left[\alpha_{i}\right]\right\|\left\|\left[\gamma_{k}\right]\right\| \|\left[x_{i j}\|\|\left[z_{k l}\right]\|\|\left[y_{i j}\right]\|\|\left[w_{k l}\right]\|\|\left[\beta_{j}\right]\|\|\left[\delta_{l}\right] \|\right.$. Hence, $\|u v\|_{s} \leq\|u\|_{s}\|v\|_{s}$ for all $u, v \in X \otimes_{s} Y$. So we may extend the product on $X \otimes_{s} Y$ to $X \otimes^{s} Y$, so that $X \otimes^{s} Y$ is a Banach algebra.

For the $*$-part, let $u \in X \otimes Y$ with $u=\alpha(x \circ y) \beta$ then $u^{*}=\beta^{*}\left(x^{*} \circ\right.$ $\left.y^{*}\right) \alpha^{*}$. So $\left\|u^{*}\right\|_{s} \leq\left\|\beta^{*}\right\|\left\|x^{*}\right\|\left\|y^{*}\right\|\left\|\alpha^{*}\right\|=\|\beta\|\|x\|\|y\|\|\alpha\|$. Thus $\left\|u^{*}\right\|_{s} \leq$ $\|u\|_{s}$. Similarly, $\|u\|_{s} \leq\left\|u^{*}\right\|_{s}$.

One can easily verify that $\|\cdot\|_{s} \leq\|\cdot\|_{\gamma}$ on $X \otimes Y$, giving that $\|\cdot\|_{s}$ is an admissible cross norm on $X \otimes Y$. Therefore, $X \otimes^{s} Y$ has a bounded approximate identity, whenever $X$ and $Y$ are approximately unital. 
In particular, for $C^{*}$-algebras $A$ and $B, A \otimes^{s} B$ is a Banach *-algebra with a bounded approximate identity, and it is a $C^{*}$-algebra if and only if either $A=\mathbb{C}$ or $B=\mathbb{C}$, which follows directly by ( [4, Corollary 5.13) and ( [2], Corollary 3). Also, ( [17], Theorem 4.8) yields that the enveloping $C^{*}$-algebra, $C^{*}\left(A \otimes^{s} B\right)$, of $A \otimes^{s} B$ is $A \otimes_{\max } B$, the maximal tensor product of $A$ and $B$.

As with the Haagerup tensor norm and the operator space projective tensor norm, we define an intimately related class of bilinear maps for the schur tensor product of operator spaces by mimicking the schur multiplication of matrices.

Definition 2.3. Given operator spaces $V, W$ and $Z$, a bilinear map $\varphi$ : $V \times W \rightarrow Z$ is said to be schur bounded bilinear map if the associated maps $\varphi_{n}: M_{n}(V) \times M_{n}(W) \rightarrow M_{n}(Z)$ given by

$$
\varphi_{n}\left(\left(v_{i j}\right),\left(w_{i j}\right)\right)=\left(\varphi\left(v_{i j}, w_{i j}\right)\right), n \in \mathbb{N}
$$

are uniformly bounded, and in this case we denote $\|\varphi\|_{s b}=\sup _{n}\left\|\varphi_{n}\right\|$.

Equivalently, a bilinear map $\varphi: V \times W \rightarrow Z$ is schur bounded if and only if the associated maps $\varphi_{n}: M_{n}(V) \times M_{n}(W) \rightarrow M_{n}(Z)$ given by

$$
\varphi_{n}(\alpha \otimes v, \beta \otimes w)=\alpha \circ \beta \otimes \varphi(v, w), \alpha, \beta \in M_{n}, v \in V, w \in W,
$$

where $\alpha \circ \beta$ is the usual schur multiplication of matrices, are uniformly bounded. Indeed, for $\alpha=\left[\alpha_{i j}\right]$ and $\beta=\left[\beta_{i j}\right], \alpha \otimes v=\left[\alpha_{i j} v\right]$ and $\beta \otimes$ $w=\left[\beta_{i j} w\right]$ by using the identification $M_{n}(V) \cong M_{n} \otimes V$. Thus, by the above definition, $\varphi_{n}(\alpha \otimes v, \beta \otimes w)=\varphi_{n}\left(\left[\alpha_{i j} v\right],\left[\beta_{i j} w\right]\right)=\left(\varphi\left(\alpha_{i j} v, \beta_{i j} w\right)\right)=$ $\left(\alpha_{i j} \beta_{i j} \varphi(v, w)\right)=\left[\alpha_{i j} \beta_{i j}\right] \otimes \varphi(v, w)=\alpha \circ \beta \otimes \varphi(v, w)$. Also, any jointly completely bounded bilinear map is schur bounded. This is immediate from the relation $\phi_{n}\left(\left[x_{i j}\right],\left[y_{i j}\right]\right)=\left(\phi\left(x_{i j}, y_{i j}\right)\right)=\alpha \phi_{(n)}\left(\left[x_{i j}\right],\left[y_{k l}\right]\right) \beta$ for $\alpha=$ $\left[e_{11}, e_{22}, \ldots, e_{n n}\right]$ and $\beta=\alpha^{t}$.

It is easy to see that $\|\cdot\|_{s b}$ is a norm on the linear space $S B(V \times W, Z)$, the space of all schur bounded bilinear maps. We now show that the schur tensor norm linearizes the schur bounded bilinear maps, that is $\left(V \otimes^{s} W\right)^{*}=$ $S B(V \times W, \mathbb{C})$.

Proposition 2.4. If $V, W$ and $X$ are operator spaces, then there is a natural isometric identification

$$
C B\left(V \otimes^{s} W, X\right)=S B(V \times W, X) .
$$

Proof: Let $\varphi: V \times W \rightarrow X$ be a schur bounded bilinear map. Then there exists a unique linear mapping $\bar{\varphi}: V \otimes W \rightarrow X$ such that $\bar{\varphi}(x \otimes y)=\varphi(x, y)$ for all $x \in V$ and $y \in W$. For $u=\alpha(v \circ w) \beta \in M_{n}(V \otimes W), \alpha \in M_{n, p}$, $v \in M_{p}(V), w \in M_{p}(W)$, and $\beta \in M_{p, n}$, we have $\bar{\varphi}_{n}(u)=\alpha \varphi_{p}\left(\left[v_{i j}\right],\left[w_{i j}\right]\right) \beta$, and so

$$
\begin{aligned}
\left\|\bar{\varphi}_{n}(u)\right\| & \leq\|\alpha\|\left\|\varphi_{p}\right\|\left\|\left[v_{i j}\right]\right\|\left\|\left[w_{i j}\right]\right\|\|\beta\|, \\
& \leq\|\varphi\|_{s b}\|\alpha\|\left\|\left[v_{i j}\right]\right\|\left\|\left[w_{i j}\right]\right\|\|\beta\| .
\end{aligned}
$$

Since the above is true for every representation of $u$, so it follows that 
$\|\bar{\varphi}\|_{c b} \leq\|\varphi\|_{s b}$. For the converse part, note that $\varphi_{p}\left(\left[v_{i j}\right],\left[w_{i j}\right]\right)=\left(\varphi\left(v_{i j}, w_{i j}\right)\right)=$ $\bar{\varphi}_{p}(v \circ w)$, for $v=\left[v_{i j}\right]$ and $w=\left[w_{i j}\right]$. So $\left\|\varphi_{p}\left(\left[v_{i j}\right],\left[w_{i j}\right]\right)\right\|=\left\|\bar{\varphi}_{p}(v \circ w)\right\| \leq$ $\|\bar{\varphi}\|_{c b}\|v \circ w\|_{s} \leq\|\bar{\varphi}\|_{c b}\|v\|\|w\|$. Hence it follows that $\|\varphi\|_{s b} \leq\|\bar{\varphi}\|_{c b}$. Also the operator $\bar{\varphi}: V \otimes_{s} W \rightarrow X$ has a unique extension to an operator $\bar{\varphi}: V \otimes^{s} W \rightarrow X$ with the same norm.

To see the surjectivity, let $\psi \in C B\left(V \otimes^{s} W, X\right)$. We can define a blinear map $\varphi: V \times W \rightarrow X$ such that $\varphi(v, w)=\psi(v \otimes w)$. Using the same calculations as done in the above paragraph just replacing $\bar{\varphi}$ by $\psi$, we obtain the required result.

The above identification yields a new formula for the schur tensor norm:

$$
\|u\|_{s}=\sup \left\{|\varphi(u)|: \varphi \in S B(V \times W, \mathbb{C}),\|\varphi\|_{s b} \leq 1\right\} .
$$

The following corollary, which shows that the schur tensor product is functorial, is a simple consequence of the above Proposition.

Corollary 2.5. Let $V, V_{1}, W$ and $W_{1}$ be operator spaces. Given the complete contractions $\varphi: V \rightarrow V_{1}$ and $\phi: W \rightarrow W_{1}$, the corresponding map $\varphi \otimes \phi: V \otimes W \rightarrow V_{1} \otimes W_{1}$ extends to a complete contraction map $\varphi \otimes^{s} \phi: V \otimes^{s} W \rightarrow V_{1} \otimes^{s} W_{1}$.

For operator spaces $V$ and $W$, let $B(V, W)$ denote the bounded linear maps from $V$ to $W$. Define the matrix norm structure on $B(V, W)$ by identifying $\left[f_{i j}\right] \in M_{n}(B(V, W))$ with the map $\left[f_{i j}\right]: M_{n}(V) \rightarrow M_{n}(W)$ defined by $\left[f_{i j}\right]\left(\left[x_{i j}\right]\right)=\left[f_{i j}\left(x_{i j}\right)\right]$. Let ${ }_{s} B(V, W)$ denote the space $B(V, W)$ with this matrix norm structure. Then we have the following:

Proposition 2.6. For operator spaces $V, W$ and $Z, S B(V \times W, Z)=$ $C B\left(V,{ }_{s} B(W, Z)\right)$, here equal sign signify the isometric isomorphism.

Proof: Let $u: V \times W \rightarrow Z$ be a schur bounded bilinear map, write $\widetilde{u}$ for the map from $V$ to the set of functions from $W$ to $Z$ defined by $\widetilde{u}(v)(w)=$ $u(v, w), v \in V$ and $w \in W$. Then $\|\widetilde{u}\|_{c b}=\sup \left\{\left\|\left[\widetilde{u}\left(v_{i j}\right)\right]\right\|_{M_{n}(s B(W, Z))}\right.$ : $\left.\left[v_{i j}\right] \in M_{n}(V)_{1}, n \in \mathbb{N}\right\}=\sup \left\{\left\|\left[\widetilde{u}\left(v_{i j}\right)\left(w_{i j}\right)\right]\right\|:\left[v_{i j}\right] \in M_{n}(V)_{1},\left[w_{i j}\right] \in\right.$ $\left.M_{n}(W)_{1}, n \in \mathbb{N}\right\}=\sup \left\{\left\|\left[u\left(v_{i j}, w_{i j}\right)\right]\right\|:\left[v_{i j}\right] \in M_{n}(V)_{1},\left[w_{i j}\right] \in M_{n}(W)_{1}, n \in\right.$ $\mathbb{N}\}=\|u\|_{s b}$. For the converse, let $v \in C B\left(V,{ }_{s} B(W, Z)\right)$ and define $u(x, y)=$ $v(x)(y)$, then reversing the last argument shows that $u$ is schur bounded.

The proof of the following propositions are essentially the same as those for the operator space projective tensor product, so we skip them.

Proposition 2.7. [Projective] Let $V, V_{1}, W$ and $W_{1}$ be operator spaces. Given the complete quotient maps $\varphi: V \rightarrow V_{1}$ and $\phi: W \rightarrow W_{1}$, the corresponding map $\varphi \otimes \phi: V \otimes W \rightarrow V_{1} \otimes W_{1}$ extends to a complete quotient $\operatorname{map} \varphi \otimes^{s} \phi: V \otimes^{s} W \rightarrow V_{1} \otimes^{s} W_{1}$.

Furthermore,

$\operatorname{ker} \varphi \otimes^{s} \phi=c l\{\operatorname{ker} \varphi \otimes W+V \otimes \operatorname{ker} \phi\}$. 
Proposition 2.8. [Symmetric] Given operator spaces $V, W$ and $Z$, we have completely isometric isomorphism:

$$
V \otimes^{s} W \stackrel{c b}{=} W \otimes^{s} V
$$

The next proposition gives the general representation of an element of the schur tensor product.

Proposition 2.9. Given operator spaces $V$ and $W$, if $u \in M_{n}\left(V \otimes^{s} W\right)$ then

$$
\|u\|_{s}=\inf \{\|\alpha\|\|x\|\|y\|\|\beta\|: u=\alpha(x \circ y) \beta\}
$$

where infimum is taken over arbitrary decompositions with $x \in M_{\infty}(V)$, $y \in M_{\infty}(W), \alpha \in M_{n, \infty}$, and $\beta \in M_{\infty, n}$.

Proof: Suppose that $u \in M_{n}\left(V \otimes^{s} W\right)$ such that $\|u\|_{s}<1$. Let $\epsilon=$ $1-\|u\|_{s}>0$. Let $u_{k} \in M_{n}(V \otimes W)$ be the sequence such that $\left\|u-u_{k}\right\| \rightarrow 0$. We may assume that $\left\|u-u_{1}\right\|<\epsilon$ and $\left\|u_{k+1}-u_{k}\right\| \leq \frac{\epsilon}{2^{k+1}}$ for all $k \in \mathbb{N}$, from which we get $u=u_{1}+\sum_{k=1}^{\infty}\left(u_{k+1}-u_{k}\right)$. Let $t_{k}=u_{k+1}-u_{k}$ and $t_{0}=u_{1}$. Then we have that $u=\sum_{k=0}^{\infty} t_{k}$. As $t_{k} \in M_{n}(V \otimes W)$, there exist $\alpha_{k} \in M_{n, p_{k}}$, $\beta \in M_{p_{k}, n}, x_{k} \in M_{p_{k}}(V)$, and $y_{k} \in M_{p_{k}}(W)$ such that $t_{k}=\alpha_{k}\left(x_{k} \circ y_{k}\right) \beta_{k}$ with $\left\|\alpha_{k}\right\|\left\|x_{k}\right\|\left\|y_{k}\right\|\left\|\beta_{k}\right\|<\left\|t_{k}\right\|+\frac{\epsilon}{2^{k+1}}$. We can assume that $\left\|x_{k}\right\|=\left\|y_{k}\right\|=$ 1 , and $\left\|\alpha_{k}\right\|=\left\|\beta_{k}\right\|<\left(\left\|t_{k}\right\|+\frac{\epsilon}{2^{k+1}}\right)^{\frac{1}{2}}$, for $k \in \mathbb{N}$, and $\left\|\alpha_{0}\right\|\left\|\beta_{0}\right\|<1-\epsilon$. Then we have $\sum_{k=0}^{\infty}\left\|\alpha_{k}\right\|\left\|\beta_{k}\right\|<1$. Now choose an increasing sequence $c_{k} \geq 1$ with $c_{k} \rightarrow \infty$ such that $\sum_{k=0}^{\infty} c_{k}\left\|\alpha_{k}\right\|\left\|\beta_{k}\right\|<1$. Put $v=\bigoplus c_{k}^{-1} v_{k} \in M_{\infty}(V)$, $w=\bigoplus c_{k}^{-1} w_{k} \in M_{\infty}(V), \alpha=\left[c_{1} \alpha_{1}, c_{2} \alpha_{2}, \cdots, c_{r} \alpha_{r} \cdots\right] \in M_{n, \infty}$, and $\beta=$ $\alpha^{t}$. We then have $u=\alpha(v \circ w) \beta$ and all $v, w, \alpha$, and $\beta$ have norm $<1$.

We define the schur bounded map from operator space $V$ to operator space $W^{*}$ by using the identification $S B\left(V, W^{*}\right)=\left(V \otimes^{s} W\right)^{*}$, i.e. $S B\left(V, W^{*}\right)$ is isometrically isomorphic to $\left(V \otimes^{s} W\right)^{*}$.

Proposition 2.10. If $\varphi$ is a schur bounded map from $V$ to $W$. Then $\varphi^{*}$ : $W^{*} \rightarrow V^{*}$ is also schur bounded with the same schur norm.

Proof: Given that $\varphi$ is a schur bounded map from $V$ to $W$, meaning that $i \circ \varphi: V \rightarrow W^{* *}$ is schur bounded, where $i: W \rightarrow W^{* *}$ is the natural embedding. Thus there exists $\phi \in\left(V \otimes^{s} W^{*}\right)^{*}$ such that $\phi(v \otimes f)=i$ 。 $\varphi(v)(f)=f(\varphi(v))$, for all $v \in V$ and $f \in W^{*}$, with $\|\phi\|=\|i \circ \varphi\|$. Now consider the map $\theta: W^{*} \otimes^{s} V \rightarrow V \otimes^{s} W^{*}$, which is completely isometric by Proposition 2.8. So $\phi \circ \theta \in\left(W^{*} \otimes^{s} V\right)^{*}$ with $\phi \circ \theta(f \otimes v)=\phi(v \otimes f)=$ $f(\varphi(v))=\varphi^{*}(f)(v)$. Thus $\|\phi \circ \theta\|=\left\|\varphi^{*}\right\|$. As $\theta$ is completely isometric 
map from $W^{*} \otimes^{s} V$ onto $V \otimes^{s} W^{*}$, so it follows that $\|\phi \circ \theta\|=\|\phi\|$. Thus $\|i \circ \varphi\|=\|\varphi\|=\left\|\varphi^{*}\right\|$.

If we use the following explicit definition of schur bounded map then it follows easily that every linear functional $f$ is schur bounded with $\|f\|_{s b}=$ $\|f\|$.

Definition 2.11. Given operator spaces $V$ and $W$, let $\varphi: V \rightarrow W^{*}$ be a linear map. We say that $\varphi$ is schur bounded map if $\|\varphi\|_{s b}=\sup \left\{\left\|\left[\varphi\left(x_{i j}\right)\left(y_{i j}\right)\right]\right\|\right.$ : $\left.\left\|\left[x_{i j}\right]\right\|_{M_{n}(V)} \leq 1,\left\|\left[y_{i j}\right]\right\|_{M_{n}(W)} \leq 1, n \in \mathbb{N}\right\}<\infty$.

Using the above definition, one can easily prove that $S B(V \times W, \mathbb{C})=$ $S B\left(V, W^{*}\right)$. Proposition 2.10 can also be proved by using this explicit definition of schur bounded maps.

We now proceed to show that the schur tensor product of two matrix ordered space is matrix ordered. Recall that a complex vector space $V$ is said to be matrix ordered if (1) $V$ is a ${ }^{*}$-vector space, (2) Each $M_{n}(V)$, $n \geq 1$, is partially ordered by a cone $M_{n}(V)_{+} \subseteq M_{n}(V)_{s a}$, the self adjoint part of $M_{n}(V)$, and (3) If $\gamma \in M_{m, n}$, then $\gamma^{*} M_{m}(V)^{+} \gamma \subseteq M_{n}(V)^{+}$. Also, by an involutive operator space, we mean an operator space with an involution such that, for each $n \in \mathbb{N}, M_{n}(V)$ is an involutive Banach space with the natural involution, i.e., the involution on $M_{n}(V)$ is an isometry.

Proposition 2.12. Let $V$ and $W$ be an involutive operator spaces. If $u \in$ $M_{n}\left(V \otimes_{s} W\right)_{s a}$ then $u$ has a representation $u=\alpha(x \circ y) \alpha^{*}$, where $\alpha \in M_{n, p}$, $x \in M_{p}(X)_{s a}$ and $y \in M_{p}(Y)_{\text {sa }}$. Moreover, $\|u\|_{s}=\inf \left\{\|\alpha\|^{2}\|\| x\|\| y \|: u=\right.$ $\left.\alpha(x \circ y) \alpha^{*}, \alpha \in M_{n, p}, x \in M_{p}(X), y \in M_{p}(Y), p \in \mathbb{N}\right\}$.

Proof: Let $u \in M_{n}\left(V \otimes_{s} W\right)_{s a}$ and $\epsilon>0$. Then there exist $\alpha \in M_{n, p}$, $\beta \in M_{n, p}, x \in M_{p}(V)$ and $y \in M_{p}(W)$ such that $u=\alpha(x \circ y) \beta$ with $\|u\|_{s} \leq\|\alpha\|\|x\|\|y\|\|\beta\| \leq\|u\|_{s}+\epsilon$. As $u$ is self adjoint, so we have

$$
\begin{aligned}
u & =\frac{1}{2}\left(u+u^{*}\right) \\
& =\frac{1}{2}\left(\alpha(x \circ y) \beta+\beta^{*}\left(x^{*} \circ y^{*}\right) \alpha^{*}\right) . \\
& =\left(\begin{array}{cc}
\frac{\lambda \beta^{*}}{\sqrt{2}} & \frac{\lambda^{-1} \alpha}{\sqrt{2}}
\end{array}\right)\left(\begin{array}{cc}
0 & x^{*} \\
x & 0
\end{array}\right) \circ\left(\begin{array}{cc}
0 & y^{*} \\
y & 0
\end{array}\right)\left(\begin{array}{c}
\frac{\lambda \beta}{\sqrt{2}} \\
\frac{\lambda^{-1} \alpha^{*}}{\sqrt{2}}
\end{array}\right)
\end{aligned}
$$

for any $\lambda>0$. Let $v:=\left(\begin{array}{cc}0 & x^{*} \\ x & 0\end{array}\right), w:=\left(\begin{array}{cc}0 & y^{*} \\ y & 0\end{array}\right)$, and $\tilde{\alpha}=\left(\begin{array}{ll}\frac{\lambda \beta^{*}}{\sqrt{2}} & \frac{\lambda^{-1} \alpha}{\sqrt{2}}\end{array}\right)$. Then we have $u=\tilde{\alpha}(v \circ w) \tilde{\alpha}^{*}$ and $\|u\|_{s} \leq\|\tilde{\alpha}\|^{2}\|v\|\|w\| \leq\left[\frac{1}{2}\left(\lambda^{2}\|\beta\|^{2}+\right.\right.$ $\left.\left.\lambda^{-2}\|\alpha\|^{2}\right)\right]\|v\|\|w\|$, where $v$ and $w$ are self adjoint elements. Now, by using the fact that $\min _{\lambda>0} \frac{1}{2}\left(\lambda^{2}\|\beta\|^{2}+\lambda^{-2}\|\alpha\|^{2}\right)=\|\beta\|\|\alpha\|$, given $\delta>0$ choose $\lambda_{0}>0$ such that $\|\beta\|\|\alpha\|+\delta>\frac{1}{2}\left(\lambda_{0}^{2}\|\beta\|^{2}+\lambda_{0}^{-2}\|\alpha\|^{2}\right)$. We then have $\|u\|_{s} \leq\|\tilde{\alpha}\|^{2}\|v\|\|w\| \leq\|\beta\|\|\alpha\|\|x\|\|y\|$. Thus, we get the desired norm condition.

Based on the above, we define $\left(V \otimes \otimes^{s} W\right)_{+}=\operatorname{cl}_{s}\left\{\alpha(v \circ w) \alpha^{*}: v \in M_{p}(V)^{+}, w \in\right.$ $\left.M_{p}(W)^{+}, \alpha \in M_{n, p}\right\}$, and we have the following, which can be proved easily. 
Proposition 2.13. For matrix ordered operator spaces $V$ and $W, V \otimes^{s} W$ is a matrix ordered operator space.

Let $X$ and $Y$ be operator spaces. For $f \in C B\left(X, M_{p}\right)$ and $g \in C B\left(Y, M_{p}\right)$, define $f \bar{\circ} g: X \otimes Y \rightarrow M_{p}$ on elementary tensor as $f \bar{\circ} g(x \otimes y)=f(x) \circ$ $g(y)$ for $x \in X$ and $y \in Y$. Define a norm on $M_{n}(X \otimes Y)$ as $\|u\|_{s^{\prime}}=$ $\sup \left\{\left\|(f \bar{\circ} g)_{n}(u)\right\|\right\}$, where the supremum is taken over all $f \in M_{p}\left(X^{*}\right)_{1}$ and $g \in M_{p}\left(Y^{*}\right)_{1}, p \in \mathbb{N}$. Let $X \otimes \otimes^{s^{\prime}} Y$ denote the completion of $X \otimes Y$ in the $\|\cdot\|_{s^{\prime}}$-tensor norm.

Proposition 2.14. For operator spaces $X$ and $Y$, the natural embedding $\theta: X \otimes \otimes^{s^{\prime}} Y \rightarrow S B\left(X^{*} \times Y^{*}, \mathbb{C}\right)$ is isometric.

Proof: The canonical map $\theta$ is determined by $\theta(x \otimes y)(f, g)=f(x) g(y)$ for $x \in X, y \in Y, f \in X^{*}$ and $g \in Y^{*}$. For any $u=\sum_{t=1}^{k} a_{t} \otimes b_{t} \in X \otimes Y$, by definition, $\|\theta(u)\|_{s b}=\sup \left\{\left\|\left[\theta(u)\left(f_{i j}, g_{i j}\right)\right]\right\|:\left[f_{i j}\right] \in M_{n}\left(X^{*}\right)_{1},\left[g_{i j}\right] \in\right.$ $\left.M_{n}\left(Y^{*}\right)_{1}, n \in \mathbb{N}\right\}=\sup \left\{\left\|\left[\sum_{t=1}^{k} f_{i j}\left(a_{t}\right) g_{i j}\left(b_{t}\right)\right]\right\|:\left[f_{i j}\right] \in M_{n}\left(X^{*}\right)_{1},\left[g_{i j}\right] \in\right.$ $\left.M_{n}\left(Y^{*}\right)_{1}, n \in \mathbb{N}\right\}=\sup \left\{\left\|\left[\left[f_{i j}\right] \overline{\mathrm{o}}\left[g_{i j}\right](u)\right]\right\|:\left[f_{i j}\right] \in M_{n}\left(X^{*}\right)_{1},\left[g_{i j}\right] \in M_{n}\left(Y^{*}\right)_{1}, n \in\right.$ $\mathbb{N}\}$, by using the identification $M_{n}\left(X^{*}\right)=C B\left(X, M_{n}\right)$, which is same as $\|u\|_{s^{\prime}}$.

Next we consider the useful variation of the last proposition, which shows that the dual of the schur tensor norm is the $\|\cdot\|_{s^{\prime}}$-norm.

Proposition 2.15. For operator spaces $X$ and $Y$, the natural embedding $\psi: X^{*} \otimes{ }^{s^{\prime}} Y^{*} \rightarrow S B(X \times Y, \mathbb{C})$ is isometric.

Proof: We have to show that $\left\|\sum_{t=1}^{k} f_{t} \otimes g_{t}\right\|_{s^{\prime}}=\sup \left\{\left\|\left[\sum_{t=1}^{k} f_{t}\left(x_{i j}\right) g_{t}\left(y_{i j}\right)\right]\right\|\right.$ : $\left.\left[x_{i j}\right] \in M_{n}(X)_{1},\left[y_{i j}\right] \in M_{n}(Y)_{1}, n \in \mathbb{N}\right\}$. By Proposition 2.14, we have an isometric map $X^{*} \otimes^{s^{\prime}} Y^{*} \rightarrow S B\left(X^{* *} \times Y^{* *}, \mathbb{C}\right)$. Therefore, the right-hand side is dominated by $\|u\|_{s^{\prime}}$ for $u=\sum_{t=1}^{k} f_{t} \otimes g_{t}$. Now let $\left[F_{i j}\right] \in M_{n}\left(X^{* *}\right)_{1}$, $\left[G_{i j}\right] \in M_{n}\left(Y^{* *}\right)_{1}$ then there exist $\widehat{x_{\lambda}} \in M_{n}(X)$ and $\widehat{y_{\nu}} \in M_{n}(Y)$ such that $\widehat{x_{\lambda}}$ converges to $\left[F_{i j}\right]$ and $\widehat{y_{\nu}}$ converges to $\left[G_{i j}\right]$ in the point-norm topology by ( 6], Proposition 4.2.5). Thus equality holds.

The identification $M_{n}(S B(X \times Y, \mathbb{C}))=S B\left(X \times Y, M_{n}\right)$ endows $S B(X \times$ $Y, \mathbb{C})$ with an operator space structure and make the map, defined in Proposition 2.15, completely isometric. Also note that, like the projective and injective norm, schur and delta norm are in perfect duality in the finite dimensional setting. 


\section{Equivalence of The Schur nORM}

Let $E \subseteq A$ be an operator subspace of a $C^{*}$-algebra $A$. Then $E$ is said to be completely complemented if there is a completely bounded (cb) projection $P$ from $A$ onto $E$. In analogy to the operator space projective tensor product, schur tensor does not respect subspaces in general but behaves well for completely complemented subspaces:

Lemma 3.1. Let E, $F$ be completely complemented subspaces of the $C^{*}$ algebras $A$ and $B$ complemented by cb projection having cb norm 1, respectively. Then $E \otimes^{s} F$ is a closed subspace of $A \otimes^{s} B$.

Proof: By an assumption, there are cb projections $P$ from $A$ onto $E$, and $Q$ from $B$ onto $F$ with $\|P\|_{c b}=1$, and $\|Q\|_{c b}=1$. Therefore, by Corollary 2.5. $P \otimes Q: A \otimes^{s} B \rightarrow E \otimes^{s} F$ is a bounded map and $\|P \otimes Q\| \leq 1$. Now, for $u \in E \otimes F, P \otimes Q(u)=u$, giving that $\|u\|_{E \otimes^{s} F} \leq\|u\|_{A \otimes^{s} B}$. Hence $E \otimes^{s} F$ is a closed subspace of $A \otimes^{s} B$.

In particular, if $E$ and $F$ are finite dimensional $C^{*}$-subalgebras of the $C^{*}$-algebras $A$ and $B$, respectively. Then $E \otimes^{s} F$ is a closed ${ }^{*}$-subalgebra of $A \otimes^{s} B$ by ( [1, II 6.10.4(iii)). Also, for von Neumann algebras $M$ and $N$, $Z(M) \otimes^{s} Z(N)$ is a closed *-subalgebra of $M \otimes^{s} N$ by ( [7], $\S 3$, Theorem C).

Recall that the tracially bounded norm on the algebraic tensor product of two $C^{*}$-algebras $A$ and $B$ is defined as

$$
\|u\|_{t b}=\inf \left\{\sum_{k=1}^{N}\left\|\left[a_{i j}^{k}\right]\right\|\left\|\left[b_{j i}^{k}\right]\right\|: u=\sum_{k=1}^{N} n^{-1} \sum_{i, j=1}^{n} a_{i j}^{k} \otimes b_{j i}^{k}\right\} .
$$

Lemma 3.2. $\|\cdot\|_{s} \leq\|\cdot\|_{t b}$ on $A \otimes B$.

Proof: Let $u=n^{-1} \sum_{k=1}^{N} \sum_{i, j=1}^{n} a_{i j}^{k} \otimes b_{j i}^{k}$, then $\|u\|_{s} \leq n^{-1} \sum_{k=1}^{N} \| \sum_{i, j=1}^{n} a_{i j}^{k} \otimes$ $b_{j i}^{k}\left\|_{s}=n^{-1} \sum_{k=1}^{N}\right\|\left(\begin{array}{llll}1 & 1 & \ldots & 1\end{array}\right)\left[a_{i j}^{k}\right] \circ\left[b_{j i}^{k}\right]\left(\begin{array}{llll}1 & 1 & \ldots & 1\end{array}\right)^{t}\left\|_{s} \leq n^{-1} \sum_{k=1}^{N}\right\|\left(\begin{array}{llll}1 & 1 & \ldots\end{array}\right) \|$ $\left\|\left[a_{i j}^{k}\right]\right\|\left\|\left[b_{j i}^{k}\right]\right\|\left\|\left(\begin{array}{llll}1 & 1 & \ldots & 1\end{array}\right)^{t}\right\|=\sum_{k=1}^{N}\left\|\left[a_{i j}^{k}\right]\right\|\left\|\left[b_{j i}^{k}\right]\right\|$, and so $\|u\|_{s} \leq\|u\|_{t b}$.

Therefore, we have the following comparison between the various tensor norms:

$\|\cdot\|_{\lambda} \leq\|\cdot\|_{s^{\prime}} \leq\|\cdot\|_{\min } \leq\|\cdot\|_{\max } \leq\|\cdot\|_{h} \leq\|\cdot\|_{\wedge} \leq\|\cdot\|_{s} \leq\|\cdot\|_{t b} \leq\|\cdot\|_{\gamma}$.

We now look at the equivalence of the schur tensor norm with these norms.

Lemma 3.3. If $n \in \mathbb{N}$ then in $M_{n} \otimes M_{n}$,

$$
\left\|\sum_{j=1}^{n} e_{j 1} \otimes e_{j 1}\right\|_{s}=n^{1 / 2}
$$


Proof: Let $\alpha$ be a $1 \times n$ matrix with 1 in the $(1,1)$ position and all other entries are zeros, and $\beta$ be a $n \times 1$ matrix with 1 in all entries. Let $x$ and $y$ be $n \times n$ matrices in $M_{n}\left(M_{n}\right)$ with first row $e_{11}, e_{21}, \ldots, e_{n 1}$, and all other entries are zeros. Now it follows, from $C^{*}$-identity and Ruan's axioms of operator space, that $\|\alpha\|=1,\|\beta\|=n^{1 / 2}$, and $\|x\|=\|y\|=1$. Since $\sum_{j=1}^{n} e_{j 1} \otimes e_{j 1}=\alpha(x \circ y) \beta$, so $\left\|\sum_{j=1}^{n} e_{j 1} \otimes e_{j 1}\right\|_{s} \leq n^{1 / 2}$. Other inequality is obvious by ( [15], Lemma 3.3(i)) and the fact that $\|\cdot\|_{\wedge} \leq\|\cdot\|_{s}$.

Lemma 3.4. Let $M$ and $N$ be von Neumann algebras and $T: M \times N \rightarrow \mathbb{C}$ be a separately normal bilinear form. Then, for each $n \in N$, the map $T_{n}$ : $M_{n}(M) \times M_{n}(N) \rightarrow M_{n}$ given by $T_{n}\left(\left(a_{i j}\right),\left(b_{i j}\right)\right)=\left(T\left(a_{i j}, b_{i j}\right)\right)$ is separately normal.

Proof: We only show that $T_{n}, n \in \mathbb{N}$ fixed, is normal in the first variable. In the second variable, result follows on the similar lines. Let $\left(a_{\lambda}\right)$ be an increasing net of positive elements in $M_{n}(M)$ such that $a_{\lambda}$ is $w^{*}$-convergent to $a \in M_{n}(M)$. Let $b=\left[b_{i j}\right]$ be fixed matrix in $M_{n}(N)$. Since $a_{\lambda} \in M_{n}(M)$, so let $a_{\lambda}=\left[a_{i j}^{\lambda}\right]$ and $a=\left[a_{i j}\right]$. Since $T$ is separately normal, so $T\left(a_{i j}^{\lambda}, b_{i j}\right)$ is $w^{*}$-convergent to $T\left(a_{i j}, b_{i j}\right)$ for each $i, j$. Thus $\left(T\left(a_{i j}^{\lambda}, b_{i j}\right)\right)$ is $w^{*}$-convergent to $\left(T\left(a_{i j}, b_{i j}\right)\right)$, showing that $T_{n}\left(a_{\lambda}, b\right)$ is $w^{*}$-convergent to $T_{n}(a, b)$.

Proposition 3.5. Let $A$ and $B$ be $C^{*}$-algebras and $\varphi: A \times B \rightarrow \mathbb{C}$ be a schur bounded bilinear form. Then there exists a unique separately normal schur bounded bilinear form $\tilde{\varphi}: A^{* *} \times B^{* *} \rightarrow \mathbb{C}$ such that $\|\varphi\|_{s b}=\|\tilde{\varphi}\|_{s b}$.

Proof: Since $\varphi: A \times B \rightarrow \mathbb{C}$ is a schur bounded bilinear form, it is in particular bounded bilinear form and thus determines a unique separately normal bilinear form $\tilde{\varphi}: A^{* *} \times B^{* *} \rightarrow \mathbb{C}$ with $\|\varphi\|=\|\tilde{\varphi}\|$ by ( [8], Corollary 2.4). We show that $\|\varphi\|_{s b}=\|\tilde{\varphi}\|_{s b}$. For $n \in \mathbb{N}$, consider the map $\tilde{\varphi}_{n}: M_{n}\left(A^{* *}\right) \times M_{n}\left(B^{* *}\right) \rightarrow M_{n}$ defined as $\tilde{\varphi}_{n}\left(\left(a_{i j}\right),\left(b_{i j}\right)\right)=\left(\tilde{\varphi}\left(a_{i j}, b_{i j}\right)\right)$. Let $a^{* *} \in M_{n}\left(A^{* *}\right)$ and $b^{* *} \in M_{n}\left(B^{* *}\right)$ with $\left\|a^{* *}\right\| \leq 1$ and $\left\|b^{* *}\right\| \leq 1$. Since the unit ball of $M_{n}(A)$ is $w^{*}$-dense in the unit ball of $M_{n}\left(A^{* *}\right)$, so we obtain a net $\left(a_{\lambda}\right)$ (resp., $\left.\left(b_{\nu}\right)\right)$ in $M_{n}(A)$ (resp., $M_{n}(B)$ ) which is $w^{*}$ convergent to $a^{* *}$ (resp., $b^{* *}$ ) with $\left\|a_{\lambda}\right\| \leq 1$ (resp., $\left\|b_{\nu}\right\| \leq 1$ ). By Lemma 3.4. $\tilde{\varphi}_{n}$ is separately normal so $\left\|\tilde{\varphi}_{n}\left(a^{* *}, b^{* *}\right)\right\| \leq \liminf _{\lambda, \nu}\left\|\tilde{\varphi}_{n}\left(\widehat{a_{\lambda}}, \widehat{b_{\nu}}\right)\right\|=$ $\liminf _{\lambda, \nu}\left\|\varphi_{n}\left(a_{\lambda}, b_{\nu}\right)\right\| \leq\left\|\varphi_{n}\right\|$. Thus $\left\|\tilde{\varphi}_{n}\right\| \leq\left\|\varphi_{n}\right\| \leq\|\varphi\|_{s b}$ for every $n \in \mathbb{N}$. Clearly, $\|\varphi\|_{s b} \leq\|\tilde{\varphi}\|_{s b}$ as $\varphi$ being the restriction of $\tilde{\varphi}$. Hence $\|\varphi\|_{s b}=\|\tilde{\varphi}\|_{s b}$.

Corollary 3.6. For $C^{*}$-algebras $A$ and $B$, the embedding $\nu=i_{A} \otimes^{s} i_{B}$ of $A \otimes^{s} B$ into $A^{* *} \otimes^{s} B^{* *}$ is an isometry.

For $C^{*}$-algebras $A$ and $B$, we know that $S B\left(A, B^{*}\right)=\left(A \otimes^{s} B\right)^{*}$. So, by Proposition 3.5, we have an isometry $\alpha:\left(A \otimes^{s} B\right)^{*} \rightarrow\left(A^{* *} \otimes^{s} B^{* *}\right)^{*}$ given by $(\alpha \phi)\left(a^{* *} \otimes b^{* *}\right)=\Phi\left(a^{* *}\right)\left(b^{* *}\right)$. Let $\omega:=\alpha^{*} \circ i: A^{* *} \otimes^{s} B^{* *} \rightarrow\left(A \otimes^{s} B\right)^{* *}$, where $i$ is the natural embedding from $A^{* *} \otimes^{s} B^{* *}$ into $\left(A^{* *} \otimes^{s} B^{* *}\right)^{* *}$. By the definition, it is clear that $\omega$ is norm reducing. 
Proposition 3.7. For $C^{*}$-algebras $A$ and $B$, we have the following:

(1) $\|\cdot\|_{s} \approx\|\cdot\|_{\gamma}$ if and only if either $A$ or $B$ is subhomogeneous.

(2) $\|\cdot\|_{s} \approx\|\cdot\|_{t b}$ if and only if either $A$ or $B$ is subhomogeneous.

(3) $\|\cdot\|_{\wedge} \approx\|\cdot\|_{t b}$ if and only if either $A$ or $B$ is subhomogeneous.

(4) $\|\cdot\|_{s} \approx\|\cdot\|_{h}$ if and only if $A$ or $B$ is finite dimensional or $A$ and $B$ are infinite dimensional subhomogeneous.

(5) $\|\cdot\|_{s} \approx\|\cdot\|_{\max }$ if and only if $A$ or $B$ is finite dimensional.

(6) $\|\cdot\|_{s} \approx\|\cdot\|_{s^{\prime}}$ if and only if $A$ or $B$ is finite dimensional.

(7) If either $A$ or $B$ is subhomogeneous then $\|\cdot\|_{s} \approx\|\cdot\|_{\wedge}$.

Proof: (1): Suppose that $A$ is subhomogeneous. Then $\|\cdot\|_{\wedge} \approx\|\cdot\|_{\gamma}$ by ( [15], Theorem 7.2), which in turn implies that $\|\cdot\|_{s} \approx\|\cdot\|_{\gamma}$.

Conversely, suppose that $\|u\|_{\gamma} \leq K\|u\|_{s}$, for some constant $K>0$, for all $u \in A \otimes B$. Since the map $\omega$ is norm reducing, so $\|\omega(u)\|_{* *} \leq\|u\|_{s}$ for all $u \in A^{* *} \otimes B^{* *}$. Also, by ( [16], Theorem 2.3), we have $\|u\|_{\gamma} \leq 2\|\mu(u)\|_{* *}$ for all $u \in A^{* *} \otimes B^{* *}$, where $\mu$ is a map from $A^{* *} \otimes^{\gamma} B^{* *}$ into $\left(A \otimes^{\gamma} B\right)^{* *}$, and $\|\cdot\|_{* *}$ denotes the relevant second dual norm. Therefore, $\|u\|_{\gamma} \leq 2\|\mu(u)\|_{*_{*}} \leq$ $2 K\|\omega(u)\|_{* *} \leq 2 K\|u\|_{s}$ for all $u \in A^{* *} \otimes B^{* *}$. Now suppose that neither $A$ nor $B$ are subhomogeneous. Then, for some positive integer $n, A^{* *}$ and $B^{* *}$ contain a copy of $M_{n}$, which by Lemma 3.1 implies that $M_{n} \otimes^{s} M_{n}$ embeds isometrically into $A^{* *} \otimes^{s} B^{* *}$. Also, $M_{n} \otimes_{\gamma} M_{n}$ embeds isometrically into $A^{* *} \otimes_{\gamma} B^{* *}$ as there is a conditional expectation from $A^{* *}$ onto $M_{n}$. By Lemma 3.3 and ( [15], Lemma 3.3), it follows that

$$
n=\left\|\sum_{j=1}^{n} e_{j 1} \otimes e_{j 1}\right\|_{\gamma} \leq 2 K\left\|\sum_{j=1}^{n} e_{j 1} \otimes e_{j 1}\right\|_{s}=2 K n^{1 / 2} .
$$

Thus one of $A^{* *}$ and $B^{* *}$ cannot contain a type $I_{n}$ factor for $n \geq 4 K^{2}$, giving that either $A$ or $B$ is subhomogeneous for $n \geq 4 K^{2}$.

(2): Suppose that $\|\cdot\|_{s} \approx\|\cdot\|_{t b}$. So, by ( [3], Theorem 1), $\|\cdot\|_{s} \approx\|\cdot\|_{\gamma}$. Thus either $A$ or $B$ is subhomogeneous by (1).

Conversely, suppose that either $A$ or $B$ is subhomogeneous. Then $\|\cdot\|_{s} \approx$ $\|\cdot\|_{\gamma}$ by $(1)$, and so $\|\cdot\|_{s} \approx\|\cdot\|_{t b}$ by $([3]$, Theorem 1$)$.

(3): follows as in (2).

(4): follows directly from ( [15], Theorem 6.1 and Theorem 7.4).

(5): Since $\|\cdot\|_{s} \approx\|\cdot\|_{\max }$ implies that $\|\cdot\|_{h} \approx\|\cdot\|_{\max }$. Hence the result follows from ( [10], Theorem). Converse is obvious.

(6): Suppose that $A$ and $B$ are infinite dimensional. Choose maximal abelian subalgebras $A_{1}$ and $B_{1}$ inside $A$ and $B$, respectively, by Zorn's Lemma. By ( [14], Exercise 4.6.12), $A_{1}$ and $B_{1}$ are infinite dimensional. Since $\|\cdot\|_{s} \approx$ $\|\cdot\|_{s^{\prime}}$, so $\|\cdot\|_{h} \approx\|\cdot\|_{s^{\prime}}$. Since $\|\cdot\|_{h}$ and $\|\cdot\|_{s^{\prime}}$ are injective, so $\|\cdot\|_{h} \approx\|\cdot\|_{s^{\prime}}$ on $A_{1} \otimes B_{1}$. As $A_{1}$ and $B_{1}$ are commutative, so $\|\cdot\|_{h} \approx\|\cdot\|_{\max }$ on $A_{1} \otimes B_{1}$. Thus $A_{1}$ or $B_{1}$ is finite dimensional by ( [10], Theorem), a contradiction. Converse is trivial.

(7) follows from (3). Note that if $A$ or $B$ is finite dimensional then $\|\cdot\|_{s} \approx\|\cdot\|_{h}$ 
by (4) which in turn implies $\|\cdot\|_{s} \approx\|\cdot\|_{\wedge}$. But in this case by ( [15], Theorem 6.1 , we have that $\|\cdot\|_{s} \approx\|\cdot\|_{\gamma}$. This implies that $A$ or $B$ is subhomogeneous, which is not true in general.

Proposition 3.7 leaves open the interesting question whether $\|\cdot\|_{s} \approx\|\cdot\|_{\wedge}$ implies $A$ or $B$ is subhomogeneous or not.

Proposition 3.8. For operator spaces $V$ and $W, \max (V) \widehat{\otimes} W \cong \max (V) \otimes^{s}$ $W$.

Proof: Since $J C B(\max (V) \times W, \mathbb{C}) \subseteq S B(\max (V) \times W, \mathbb{C}) \subseteq B(\max (V) \times$ $W, \mathbb{C})$, so $([6], \S 3.3 .9)$ implies that $J C B(\max (V) \times W, \mathbb{C})=S B(\max (V) \times$ $W, \mathbb{C})$, and hence the result.

We now summarize the norms of various elements in $M_{n} \otimes M_{n}$, the calculation of which can be carried out as in ( [15], Lemma 3.1) and Lemma 3.3. The details are left to the reader.

\begin{tabular}{|r|r|r|r|r|r|r|}
\hline Elements & $\|\cdot\|_{\min }$ & $\|\cdot\|_{h}$ & $\|\cdot\|_{\wedge}$ & $\|\cdot\|_{s}$ & $\|\cdot\|_{t b}$ & $\|\cdot\|_{\gamma}$ \\
\hline$\sum_{j=1}^{n} e_{1 j} \otimes e_{j j}$ & 1 & $\sqrt{n}$ & $\sqrt{n}$ & $\sqrt{n}$ & $\sqrt{n}$ & $\sqrt{n}$ \\
\hline$\sum_{j=1}^{n} e_{j 1} \otimes e_{j j}$ & 1 & 1 & $\sqrt{n}$ & $\sqrt{n}$ & $\sqrt{n}$ & $\sqrt{n}$ \\
\hline$\sum_{j=1}^{n} e_{1 j} \otimes e_{1 j}$ & $\sqrt{n}$ & $\sqrt{n}$ & $\sqrt{n}$ & $\sqrt{n}$ & $n$ & $n$ \\
\hline$\sum_{j=1}^{n} e_{j 1} \otimes e_{j 1}$ & $\sqrt{n}$ & $\sqrt{n}$ & $\sqrt{n}$ & $\sqrt{n}$ & $n$ & $n$ \\
\hline$\sum_{i, j=1}^{n} e_{i j} \otimes e_{i j}$ & $n$ & $n$ & $n$ & $n$ & $n$ & $n$ \\
\hline$\sum_{i, j=1}^{n} e_{i 1} \otimes e_{i j}$ & $n$ & $n$ & $n$ & $n$ & $n^{\frac{3}{2}}$ & $n^{\frac{3}{2}}$ \\
\hline$\sum_{i, j=1}^{n} e_{i i} \otimes e_{i j}$ & $\sqrt{n}$ & $n$ & $n$ & $n$ & $n$ & $n$ \\
\hline$\sum_{j=1}^{n} e_{j i} \otimes e_{i j}$ & 1 & $n$ & $n$ & $n$ & $n$ & $n$ \\
\hline$\sum_{j=1}^{n} e_{1 j} \otimes e_{j 1}$ & 1 & $n$ & $n$ & $n$ & $n$ & $n$ \\
\hline$\sum_{i, j=1}^{n} e_{j j} \otimes e_{i j}$ & $\sqrt{n}$ & $\sqrt{n}$ & $n$ & $n$ & $n$ & $n$ \\
\hline$\sum_{j=1}^{n} e_{1 j} \otimes e_{i j}$ & $n$ & $n$ & $n$ & $n$ & $n^{\frac{3}{2}}$ & $n^{\frac{3}{2}}$ \\
\hline
\end{tabular}




\begin{tabular}{|r|r|r|r|r|r|r|}
\hline Elements & $\|\cdot\|_{\min }$ & $\|\cdot\|_{h}$ & $\|\cdot\|_{\wedge}$ & $\|\cdot\|_{s}$ & $\|\cdot\|_{t b}$ & $\|\cdot\|_{\gamma}$ \\
\hline$\sum_{j=1}^{n} e_{1 j} \otimes e_{j i}$ & $\sqrt{n}$ & $n^{\frac{3}{2}}$ & $n^{\frac{3}{2}}$ & $n^{\frac{3}{2}}$ & $n^{\frac{3}{2}}$ & $n^{\frac{3}{2}}$ \\
\hline$\sum_{j=1}^{n} e_{j} \otimes e_{j j} \in l_{n}^{\infty} \otimes M_{n}$ & 1 & $\sqrt{n}$ & $\sqrt{n}$ & $\sqrt{n}$ & $\sqrt{n}$ & $\sqrt{n}$ \\
\hline
\end{tabular}

\section{REFERENCES}

[1] Blackadar, B. , Operator algebras: Theory of $C^{*}$-algebras and von Neumann algebras, Springer-Verlag Berlin Heidelberg, 2006.

[2] Blecher, D. P., Geometry of the tensor product of $C^{*}$-algebras, Math. Proc. Camb. Phil. Soc. 104 (1988), 119-127.

[3] Blecher, D. P., Tracially completely bounded multilinear maps on $C^{*}$-algebras, $J$. London Math. Soc. s2-39 (1989), 514-524.

[4] Blecher, D. P. and Paulsen,V. I., Tensor Products of operator Spaces, J. Func. Anal. 99 (1991), 262-292.

[5] Effros, E.G. and Ruan, Z.-J., A new approach to opertor spaces, Canad. Math. Bull. 34 (1991), 329-337.

[6] Effros, E. G. and Ruan, Z. J., Operator spaces, Claredon Press-Oxford, 2000.

[7] Ge., L. and Kadison, R., On tensor product of von Neumann algebras, Invent. math 123 (1996),453-466.

[8] Haagerup, U., The Grothendieck inequality for bilinear forms on $C^{*}$-algebras, $A d v$. Math. 56 (1985), 93-116.

[9] Itoh, T., On the completely bounded maps of a $C^{*}$ algebra to its dual space, Bull. London Math. Soc. (1987) 19, 546-550.

[10] Itoh, T., The maximal $C^{*}$-norm and the Haagerup norm, Math. Proc. Camb. Phil. Soc. 107 (1990), 109-114.

[11] Haagerup, U. and Itoh, T., Grothendieck type norms for bilinear forms on $C^{*}$ algebras, J. Operator Theory 34 (1995), 263-283.

[12] Itoh, T, Completely positive decompositions from duals of $C^{*}$-algebras to von Neumann algebras, Math. Japonica 51 (2000), 89-98.

[13] Jain, R. and Kumar, A., Operator space projective tensor product: Embedding into second dual and ideal structure, To appear in Proc. Edin. Math. Soc, Available on arXiv:1106.2644 1 [math.OA].

[14] Kadison, R. V. and Ringrose, J. R., Fundamentals of the theory of operator algebras I. Academic Press, 1983.

[15] Kumar, A. and Sinclair, A. M., Equivalence of norms on operator space tensor products of $C^{*}$-algebras, Trans. Amer. Math. Soc. 350 (1998), 2033-2048.

[16] Kumar, A. and Rajpal, V., Projective tensor product of $C^{*}$-algebras, Available on arXiv:1305.0791 1 [math.OA].

[17] Laursen, Kjeld B., Tensor products of Banach algebras with involution, Trans. Amer. Math. Soc. 136 (1969), 467-487.

Department of Mathematics, University of Delhi, Delhi, India.

E-mail address: vandanarajpal.math@gmail.com

Department of Mathematics, University of Delhi, Delhi, India.

E-mail address: akumar@maths.du.ac.in

Department of Mathematics, University of Gunma, Gunma, Japan.

E-mail address: itoh@gunma-u.ac.jp 